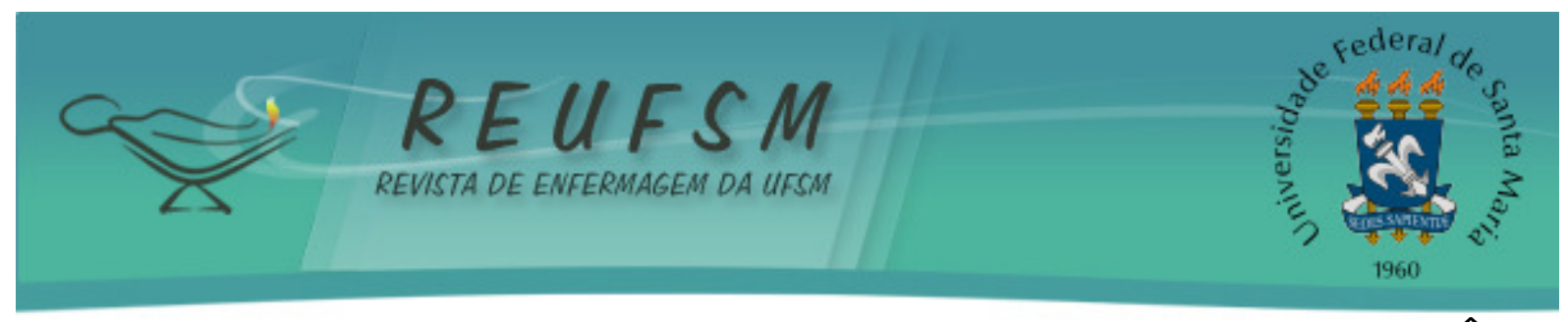

RELATO DE EXPERIÊNCIA

\title{
RODAS DE SONHO E IMAGINAÇÃO: CONTANDO HISTÓRIAS EM UM SERVIÇO DE QUIMIOTERAPIA INFANTIL
}

\section{WHEELS OF DREAMS AND IMAGINATION: TELLING STORIES IN A SERVICE OF INFANT CHEMOTHERAPY}

\section{RUEDAS DE SUEÑOS E IMAGINACIÓN: CONTANDO HISTORIAS EN EL SERVICIO DE QUIMIOTERAPIA INFANTIL}

\author{
Silmara de Oliveira Silva ${ }^{1}$ \\ Gilvânia Smith da Nóbrega Morais² \\ Joseane Almeida Dias ${ }^{3}$ \\ Suzana Pereira Cardoso da Silva ${ }^{4}$ \\ Maria Smith Pereira ${ }^{5}$ \\ Mayra Shamara Silva Batista ${ }^{6}$
}

Doi: $10.5902 / 2179769220939$

RESUMO: Objetivo: relatar a experiência de acadêmicos de enfermagem mediante 0 ato de contar histórias para crianças em tratamento oncológico. Metodologia: trata-se de um relato de experiência, do tipo descritivo, desenvolvido mediante a realização do projeto de extensão: "Rodas de sonho e imaginação: contando histórias em um serviço de quimioterapia ambulatorial infantil", que teve vigência de Maio a Dezembro de 2014, desenvolvido pela Universidade Federal de Campina Grande (UFCG-PB), em um Hospital Universitário.Resultados: por meio da contação é possível compreender aspectos relacionados ao processo de adoecimento, vivenciados pelo binômio crianças-familiares e, desse modo, transformar a prática do cuidado e tratamento. 0 estudo destaca as práticas lúdicas como principal ferramenta de aproximação entre os profissionais de saúde e o universo das crianças. Considerações Finais: ressalta-se a necessidade dos profissionais desenvolverem ações que vão ao encontro do ser criança, não se limitando a projetos que são desenvolvidos nas universidades.

Descritores: Cuidado da criança; Enfermagem oncológica; Oncologia; Imaginação; Humanização da assistência.

ABSTRACT: Aim: to report the experience of Nursing students of telling stories to children in oncologic treatment. Methodology: this is a descriptive experience report, developed from the extension project: "Wheels of dreams and imagination: telling stories at a child outpatient in chemotherapy service", from May to December 2014, developed by the Federal University of Campina Grande (UFCG-PB), at a University Hospital. Results: through storytelling, it is possible to understand aspects related to the illness process experienced by the children and their family and, thereby, transform the practice of care and treatment. The study highlights the playful practices as the main approach tool

\footnotetext{
${ }^{1}$ Enfermeira. Residente em cancerologia no Instituto de Medicina Integral Professor Fernando Figueira (IMIP) PE. Recife-PE/Brasil. Email: silmara_olyveira@hotmail.com

2 Enfermeira. Doutoranda. Docente de enfermagem da Universidade Federal de Campina Grande/UFCGPB.Brasil. Email: gilvaniamorais.ufcg@gmail.com

3 Enfermeira. Universidade Federal de Campina Grande/UFCG-PB. Brasil. Email: joseana_almeida@hotmail.com

${ }^{4}$ Enfermeira. Universidade Federal de Campina Grande/UFCG-PB. Brasil. Email: suzanapereira02@gmail.com

5 Acadêmica de psicologia. Universidade Federal de Campina Grande/UFCG-PB.Brasil. Email: smith_pereira6106@hotmail.com

6 Acadêmica de psicologia. Universidade Federal de Campina Grande/UFCG-PB.Brasil. Email: mayra.silva.ps@gmail.com
} 


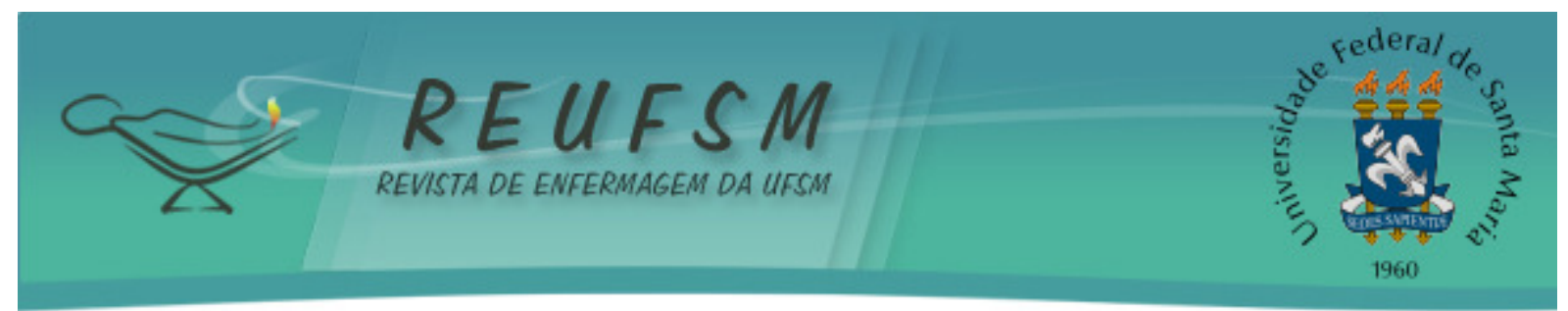

among health professionals and the children's universe. Final Thoughts: the need for professionals to develop actions that come upon the status of being a child, not getting limited by projects that are developed in universities, is emphasized.

Keywords: Child care; Oncology nursing; Medical oncology; Imagination; Humanization of assistance.

RESUMEN: Objetivo: Presentar la experiencia de estudiantes de enfermería de narración de cuentos para niños en tratamiento del cáncer. Metodología: se trata de un relato de experiencia, descriptivo, desarrollado por el proyecto de extensión: "Ruedas de sueños e imaginación: contando historias en el servicio de ambulatorio de quimioterapia infantil", entre mayo y diciembre de 2014, desarrollado por la Universidad Federal de Campina Grande (UFCG-PB), en un hospital universitario. Resultados: por medio de la historia se puede entender aspectos relacionados al proceso de la enfermedad experimentado por parte del niño y de su familia para transformar la práctica de atención. El estudio pone en relieve el enfoque de las prácticas lúdicas como la principal herramienta de los profesionales de salud para el trabajo con los niños. Consideraciones finales: se resalta la necesidad de desarrollar acciones orientadas hacia al sujeto niño, no limitándose a los proyectos que se desarrollan en las universidades.

Palabras clave: Cuidado del niños; Enfermería oncologica; Oncología médica; Imaginación; Humanización de la atención.

\section{INTRODUÇÃO}

Navegar faz parte da fantasia humana e, na criança, gera uma sensação mágica e de encantamento, típica do universo infantil. Esse encontro com a singularidade das crianças facilita a expressão de sentimentos que podem ser despertados pela contação de histórias, uma vez que, ao ouvi-las e compartilha-las, a imaginação e sonhos são ressaltados, contribuindo para o desenvolvimento cognitivo das crianças. ${ }^{1}$

Sob este prisma, ressalta-se a contação de histórias como uma arte e, como tal, exige dedicação, estudo e comprometimento, para que, assim, seja possível transmitir com total veracidade o que vai além do imaginário. Essa abordagem lúdica oportuniza a abertura de caminhos para um mundo em que os sonhos e as histórias sejam possíveis de acontecer. ${ }^{2}$

Durante a hospitalização infantil, além de descontração e relaxamento, narrar histórias exerce um importante papel no aprendizado, haja vista que aproxima a criança do universo escolar que, na maioria das vezes, devido aos procedimentos e terapêutica prolongada, acaba sendo afastado.Ademais, para as crianças com enfermidades graves, contar histórias proporciona um novo desejo de conhecer e, consequentemente, de viver, e possibilita às crianças se identificarem ou vivenciarem experiências dos personagens, além de permitirem transformar o cuidado..$^{3-4}$

Nesse panorama, encontram-se as crianças com câncer - doença crônica que demanda tratamento prolongado. Para elas, a importância de momentos de contação de histórias é essencial, pois transforma o ambiente hospitalar em um local em que possam lembrar não de procedimentos dolorosos, mas de um cuidar que é transmitido mediante a aproximação com seu universo. ${ }^{5}$

O processo de hospitalização promove, na criança em tratamento oncológico, uma quebra em sua rotina, transformando sua forma de viver, e, na maioria das vezes, ocasiona sentimentos de medo e ansiedade frente às novas mudanças. 0 profissional de enfermagem tem um papel fundamental no cuidar à criança em tratamento quimioterápico, sendo preciso que se insira no universo infantil para facilitar a adesão das crianças ao 


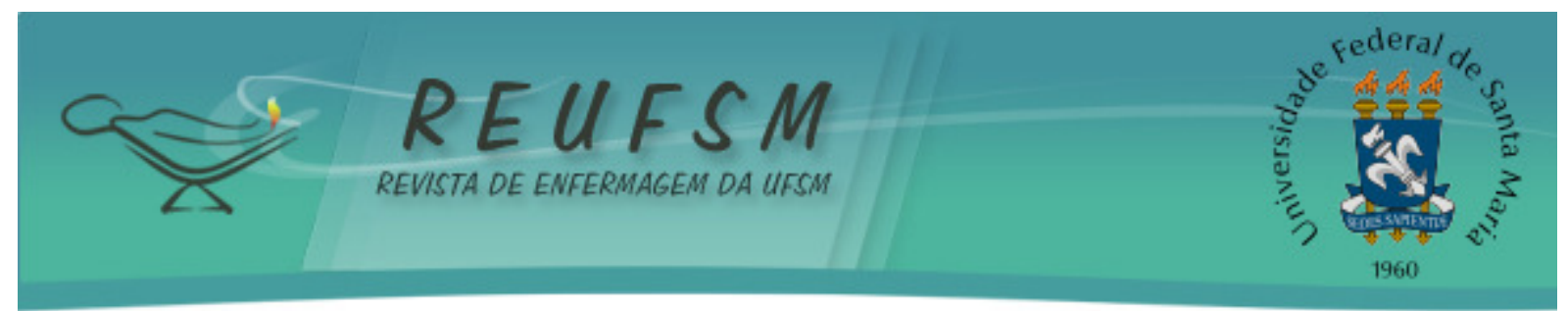

tratamento, utilizando-se de recursos lúdicos e de estratégias que permitam o acolhimento da criança. ${ }^{6}$

A inserção dos profissionais de saúde no universo infantil deve ser estimulada desde a graduação. É nesse sentido que os acadêmicos de enfermagem devem ser incentivados a participarem de projetos que visem à humanização da assistência, utilizando recursos lúdicos para aproximação do universo infantil, e reconhecendo a importância de um cuidar humanizado. ${ }^{7}$

Cônscio da importância da narração de contos infantis, da inserção de acadêmicos na construção de ações lúdicas e levando em consideração os princípios da humanização em saúde, foi desenvolvido em um serviço de quimioterapia ambulatorial infantil o projeto de extensão "Rodas de sonho e imaginação: contando histórias em um serviço de quimioterapia ambulatorial infantil", que resultou em vivências que merecem ser compartilhadas. Sob este prisma, o estudo parte da seguinte questão: qual a vivência de acadêmicos de enfermagem acerca do ato de contar histórias para crianças em tratamento oncológico?

Assim, o presente estudo tem como objetivo relatar a experiência de acadêmicos de enfermagem mediante 0 ato de contar histórias para crianças em tratamento oncológico.

\section{METODOLOGIA}

Trata-se de um relato de experiência, que se constitui como um tipo de estudo descritivo, que busca apresentar uma reflexão acerca de uma ação vivenciada, sendo de fundamental importância para o meio acadêmico. ${ }^{8}$

0 estudo envolveu a vivência de acadêmicos de enfermagem, integrantes do projeto de extensão "Rodas de sonho e imaginação: contando histórias em um serviço de quimioterapia ambulatorial infantil”, que teve vigência de Maio a Dezembro de 2014, desenvolvido pela Universidade Federal de Campina Grande (UFCG-PB), em um Hospital Universitário.

No que diz respeito ao cenário do estudo - o serviço de quimioterapia ambulatorial infantil - em que foram implementadas as atividades extensionistas de contação de história, estão em tratamento um total de 40 usuários, incluindo crianças e adolescentes. Trabalha no setor uma equipe multiprofissional, composta por enfermeiros, médicos, psicólogos, nutricionistas e fisioterapeutas, além de ser campo de estágio para discentes dos cursos da área de saúde da UFCG.

Vale ressaltar que participaram do projeto 21 integrantes, incluindo enfermeiras e acadêmicos de medicina, enfermagem e psicologia que, para operacionalizar o projeto, foram divididos em subgrupos.

Para o desenvolvimento do projeto, inicialmente, foram planejadas capacitações com os acadêmicos por meio de oficinas lúdicas com uma contadora de histórias, juntamente com a coordenadora do projeto, em que era possível trabalhar o universo da criança com câncer. Posteriormente, realizaram-se oficinas para confeccionar fantoches que seriam utilizados no projeto para a realização das contações. Após esse processo de preparação, que perdurou por três meses, foi possível os acadêmicos atuarem em campo.

No que se refere a este relato de experiência, foi descrita a vivência de uma ação desenvolvida no dia 10 de setembro de 2014, no ambulatório de quimioterapia infantil, e que foi denominada: “Como um barco navegando eu vou [...]", remetendo à ação desenvolvida por meio da utilização de barcos de papéis como recurso lúdico. Participaram dessa atividade três acadêmicos de enfermagem, 15 crianças que estavam no ambulatório de quimioterapia, juntamente com seu responsável, uma técnica de enfermagem, uma 


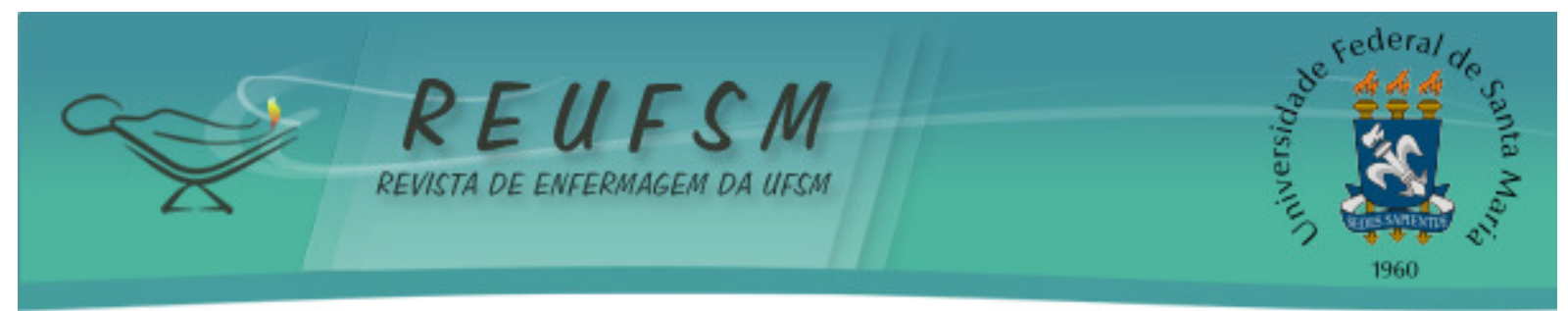

enfermeira e uma psicóloga, que estava na assistência no dia da atividade. A ação foi desenvolvida mediante a confecção de barcos de papéis e a formulação de uma história construída pelas crianças e responsáveis baseada na confecção dos barcos.

Utilizaram-se, para coleta de dados, informações presentes em diário de campo, associadas à observação participante. E, para uma melhor compreensão da experiência vivenciada, esta foi discutida em quatro seções, a saber: 1) Descrição da atividade implementada - Navegando ao encontro do universo das crianças; 2) Contação de história e familiares; 3) A função terapêutica da arte de contar histórias para as crianças oncológicas, 4) Relatando a vivência dos acadêmicos.

Para a construção do artigo, consideraram-se os preceitos legais da resolução $n^{\circ} 466 / 2012$ e do Código de Ética em Enfermagem, resolução $n^{\circ} 311 / 2007$. Por se tratar de um relato de experiência, não se faz necessária a certificação pelo Comitê de Ética em Seres Humanos.

\section{RESULTADOS E DISCUSSÃO}

\section{Navegando ao encontro do universo das crianças}

O primeiro contato com as crianças foi por meio de um barco de papel como recurso lúdico, motivados pela simples proposta de confeccionar barquinhos de papel, colorir conforme a criatividade e construir uma história. Elas acolheram com entusiasmo e transformaram o encontro em algo mágico e gratificante.

Participaram da atividade 15 crianças, a maioria utilizou cores vibrantes para pintar seus barcos, todavia uma criança com idade aproximada de 12 anos pintou seu barco todo de preto. Era sua primeira consulta no ambulatório de quimioterapia e sua expressão era de raiva, angústia e incerteza, sensações relacionadas à cor que, por vezes, simboliza morte. ${ }^{9}$

Observa-se que, muitas vezes, quando a criança recorda os momentos da doença,a vida perde um pouco a cor, demonstrando, assim, a importância das ações humanizadas e a necessidade do diálogo. Desta forma, o desenho, no contexto hospitalar, facilita a expressão infantil em situações de inibição, quando se solicita à criança, por exemplo, que desenhe o que gosta e o que não gosta no hospital, a fim de captar possíveis conflitos projetados nos desenhos, o que auxilia no esclarecimento e na elaboração de intervenções. $^{2-9}$

Sob este prisma, os pacientes oncológicos expressam sentimentos de angústia, depressão ou outros problemas psicológicos quando saem de sua rotina e têm de assumir outra forma de viver, que inclui pessoas e ambientes desconhecidos, como são os hospitais no primeiro contato, sendo demonstrados por meio de seus atos. Sendo assim, as atividades lúdicas desenvolvidas no ambiente hospitalar contribuem, sobremaneira, para que as crianças expressem sentimentos decorrentes do processo de adoecimento vivenciado. ${ }^{10}$

Ademais, alguns estudos ressaltam a importância da utilização de desenhos para identificar e minimizar o impacto da doença em crianças e adolescentes. Dessa forma, os profissionais de saúde podem acompanhar o processo de aceitação da criança e familiares acerca do tratamento oncológico utilizando recursos didáticos que facilitem a integração ${ }^{11}$.

Dando continuidade à descrição da atividade implementada, após a confecção dos barquinhos de papel, solicitou-se que os participantes construíssem uma história utilizando como personagens os barcos. Os pais e crianças foram montando uma história para cada barquinho e por meio da observação participante foi possível montar uma história coletiva, 


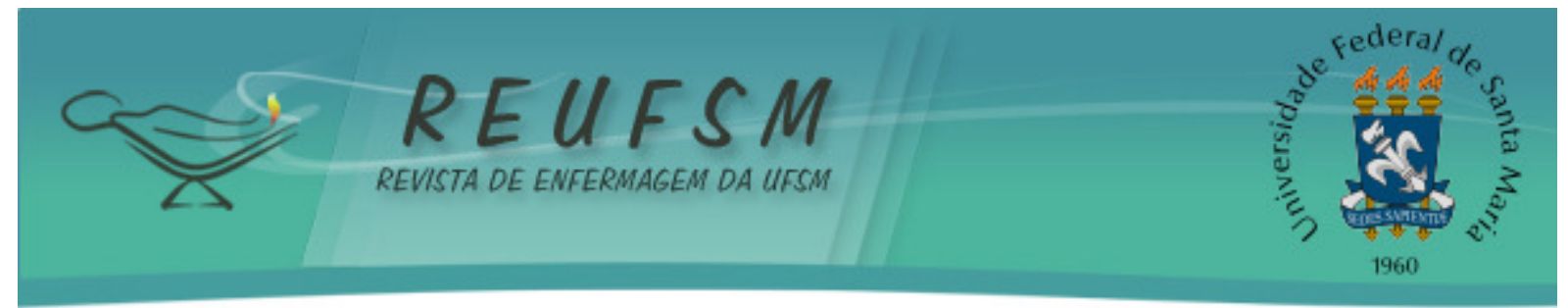

que posteriormente foi narrada por meio do processo de contação pelos integrantes do projeto para as crianças e pais que estavam participando da atividade.

A história elaborada coletivamente descreve o encontro de barcos até então desconhecidos, que se ajudam mutuamente, com a intenção de retomar, cada um, seu caminho. É assim que as crianças e pais percebem o ambulatório, especialmente na primeira consulta. Tudo é estranho, desconhecido, mas, ao longo do percurso, vínculos vão sendo construídos, vivências e histórias vão sendo compartilhadas, permitindo um enfrentamento satisfatório da situação vivenciada.

Percebeu-se, por meio da história construída, como a criatividade de crianças e pais, a partir de uma atividade lúdica, pode colaborar para que os profissionais de saúde compreendam aspectos relacionados ao processo de adoecimento, vivenciados pelo binômio e, desse modo, transformem a prática do cuidado. Sendo assim, ressalta-se a importância dos profissionais que trabalham em setores destinados ao tratamento oncológico executarem na prática ações que vão ao encontro do universo infantil.

Em um momento da construção da História, as crianças falam de tempestade, desanimação, tristeza, porém aparece alguém que faz ressurgir uma nova esperança e ajuda o barquinho a reencontrar seu amigo. A vida das crianças em tratamento oncológico é conturbada e inclui momentos de superação, desafios e sonhos, sendo essenciais momentos que forneçam suporte para a expressão de sentimentos, contribuindo, assim, para uma assistência de qualidade.

\section{Contação de história e familiares}

No momento da construção da narrativa, os pais colaboraram confeccionando o barquinho de papel, desenhando, e até mesmo narrando trechos da história, expressando verbalmente a satisfação em participar da atividade. Esse contentamento demonstrado pelos familiares se deve ao fato de a atividade lúdica, além de ser uma fonte de lazer, reforçar laços familiares e contribuir para que sentimentos sejam expressos. ${ }^{12}$

Analisando os sentimentos gerados pelas atividades lúdicas durante o período de internação de pacientes oncológicos, observa-se que as atividades proporcionam, aos participantes, alegrias, tornando o ambiente mais acolhedor e trazendo sensações de bem estar. Isso é importante para a terapêutica das crianças, pois ameniza o sofrimento e contribui para o fortalecimento dos pais. ${ }^{13}$

Portanto, o uso do lúdico no ambiente hospitalar é importante, e deve ser considerada, pelos profissionais e pela família, a maneira mais adequada de se aproximar da criança, uma estratégia capaz de desenvolver uma empatia entre ambos, de ver e compreender o mundo com os olhos da criança, estabelecendo vínculos de amizade, amor e confiança entre profissional, criança e família. ${ }^{14}$

\section{A função terapêutica da arte de contar histórias para as crianças oncológicas}

O cuidado em oncologia pediátrica não se limita à realização de procedimentos. Inclui, sobretudo, o componente emocional, o aspecto cognitivo, a percepção, o conhecimento e, inclusive, a intuição, desenvolvendo habilidade para ajudar as crianças e seus familiares a encontrarem os seus potenciais, e lidarem com as adversidades. ${ }^{15}$

Assim sendo, as histórias narradas para crianças oncológicas proporcionam um momento de descontração e relaxamento, como também contribuem para o bem-estar dos pequenos. Além disso, exercem um importante papel no que tange ao aspecto pedagógico, por integrar a criança às atividades escolares. ${ }^{16}$ 


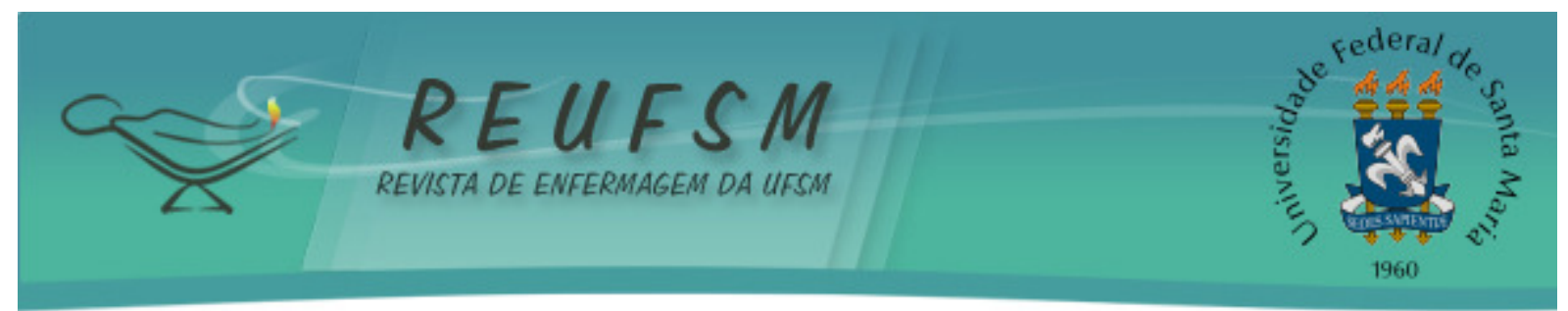

Estudodestaca que dentre os benefícios de contar histórias estão a melhor aceitabilidade de procedimentos dolorosos, alívio da dor, esquecimento da doença, sentimentos de alegria, relaxamento e confiança, melhora da autoestima, melhora da doença, viagem ao mundo da fantasia, construção do processo de leitura/hábito à leitura, resgate do sonho e do imaginário. ${ }^{17}$

Desse modo, mediante a contação de histórias é possível vivenciar um cuidar humanizado, em que é possível transformar, criar, compartilhar e realmente cuidar, diante da intercalação de ações que visam atender às necessidades do ser criança. Assim, as histórias podem acelerar a recuperação da criança e possibilitar sua interação com os adultos e profissionais da saúde.

\section{Relatando a vivência dos acadêmicos}

A experiência de estar com as crianças despertou o lado mais bonito e feliz de ver e viver a vida. Percebe-se que ser criança é demonstrar toda a essência do seu ser, é transformar o viver, é dar e receber, é sorrir sem querer, é a espontaneidade de transformar cada amanhecer.

A partir da aproximação com cada criança, foi possível refletir e desabrochar os sentimentos mais profundos, os quais proporcionaram momentos de alegria, de sorrisos, lágrimas, mas de sensação de estar mais perto do que era desejado na preparação do projeto. Também, de intervir no serviço, propiciando às crianças ações que ajudassem a vivenciar a vida de uma forma mais leve, de flutuar mediante a realidade, mesmo passando por determinados desafios, mas tendo um momento em que se podia esquecer um pouco o que cada um estava passando e aprender com o próximo.

Dessa maneira, a presença do lúdico funciona como elo entre a criança e os profissionais de saúde, caracterizando-se como um recurso que tem por finalidade facilitar ou conduzir aos objetivos estabelecidos. Além disso, o uso do lúdico, principalmente a contação de histórias, funciona como veículo de comunicação entre a criança e o profissional, tornando a relação entre eles mais produtiva, oferecendo à criança mais oportunidades de participar efetivamente do seu tratamento. ${ }^{12}$

Por meio desse relato de experiência, foi possível demonstrar as vivências compartilhadas e destacar a necessidade de implantar ações desse porte em hospitais que fornecem tratamento oncológico infantil. A experiência foi exitosa por se tratar da contação de histórias realizada por acadêmicos em um ambulatório de quimioterapia, uma vez que, em muitos casos, esta atividade é realizada em sala de aula ou eventos.

A enfermagem, na jornada da arte de cuidar, desempenha uma tarefa árdua, na qual precisa acolher o ser humano em sua essência. Assim, as crianças, como seres de luz, precisam ser integradas na simplicidade com que transparecem.

\section{CONSIDERAÇÕES FINAIS}

A partir do projeto "Rodas de sonho e imaginação", percebeu-se o quanto contar histórias e a prática de atividades lúdicas inspiram as crianças em tratamento contra o câncer. A contação não só desenvolve a imaginação e a criatividade, como também pode, por meio das vivências dos personagens, desenvolver meios de lidar com seus problemas e dificuldades do dia a dia.

A história pode transformar a vida da criança, auxiliando-a no caminho para suas descobertas com significado, pois contar histórias pode abrir as portas de um novo mundo e para visões de imenso prazer, transformando e se deixando transformar por meio da imaginação. Desse modo, o projeto "Rodas de sonho e imaginação" proporcionou às 


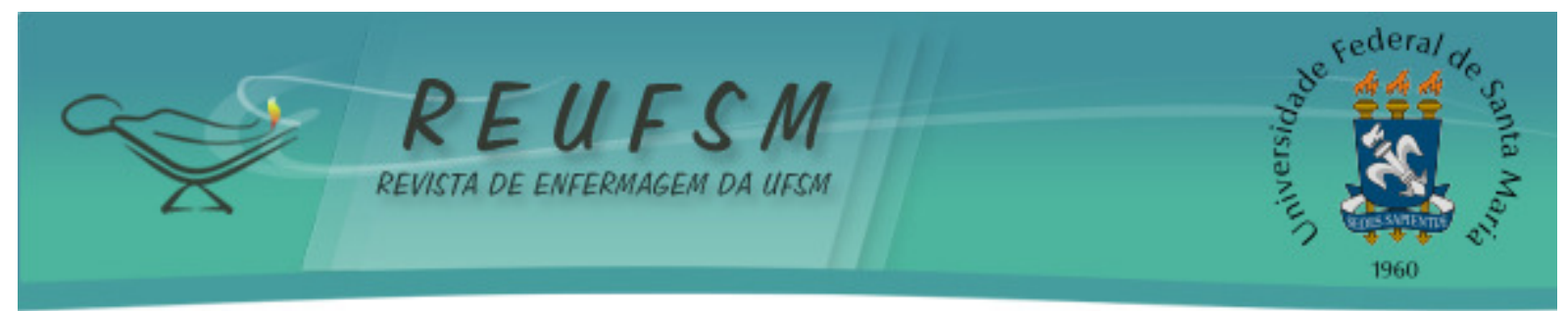

crianças, durante os momentos de contação, uma viagem ao imaginário, promovendo alento, alívio das tensões e ansiedades, fatores que colaboram no enfrentamento da situação vivenciada.

No que diz respeito às implicações para novas pesquisas na área, destaca-se a necessidade de incentivar projetos lúdicos que facilitem o cuidar para cada ser humano mediante suas reais necessidades. Compreende-se, assim, que as crianças precisam ser respeitadas em sua essência, e os profissionais, cada vez mais, integrados nesse universo e desempenhando suas ações com eficiência e satisfação para os pacientes.

É preciso incentivar a prática de contar histórias, ou outras ações lúdicas, no ambiente hospitalar, e que essas sejam realizadas pelos profissionais da própria instituição, para que não fique limitada aos projetos que são desenvolvidos.

Esse estudo contribui para que os profissionais de saúde, principalmente a equipe de enfermagem e acadêmicos, possam refletir sobre suas atividades no cenário da oncologia infantil.

\section{REFERÊNCIAS}

1. Neder DLSM, Almeida ECS, Cunha LAL, Fernandes LCS, Castro TL, Almeida TC, et al. A importância da contação de histórias como prática educativa no cotidiano escolar. Pedagodia em Ação [Internet]. 2009 jan-jun [acesso em 2015 jun 1];(1):61-4. Disponível em: http://periodicos.pucminas.br/index.php/pedagogiacao/article/view/648/662.

2. Otte MW, Kovács A. A magia de contar histórias. Santa Catarina: Instituto Catarinense de Pós-Graduação (ICPG); 2003.

3. Araujo AMC, Pola CO, Gouvêa ME, Rosa IPR. A arte de contar histórias com o livro de folhas soltas: uma experiência junto a crianças em leitos hospitalares. In: IX Congresso Nacional de Educação - EDUCERE/III Encontro Sul Brasileiro de Psicopedagogia; 2009 out 26-29; Paraná. Paraná: PUCPR; 2009. p. 10293-9.

4. Bernardino MCR, Elliott AG, Rolim Neto ML. Biblioterapia com crianças com câncer. Inf Inf [Internet]. 2012 set [acesso em 2015 jul 1];17(3):198-210. Disponíve em: http://www.uel.br/revistas/uel/index.php/informacao/article/view/10992. Doi: 10.5433/1981-8920.2012v17n3p198.

5. Souza LO, Bernardino AD. A contação de histórias como estratégia pedagógica na educação infantil e ensino fundamental. Rev Educere. 2011;6(12):235-49.

6. Noda TY. As contribuições do brincar para as crianças hospitalizadas com câncer [monografia]. Maringá: Universidade Estadual de Maringá; 2012. 30 p.

7. Souza Neto VL, Costa MADJ, Silva RAR, Santos NCCB. Ações lúdicas como ferramenta para prevenção da obesidade do pré-escolar: relato de experiência. Rev Enferm UFSM. 2014 out/dez;4(4):850-7.

8. Cavalcante BLL, Lima UTS. Relato de experiência de uma estudante de enfermagem em um consultório especializado em tratamento de feridas. J Nurs Health. 2012;1(2):94-103.

9. Freitas AKM. Psicodinâmica das cores em comunicação. Nucon-Núcleo de Comunicação. 2007;4(12):18-2.

10. Brito NTG, Carvalho R. A humanização segundo pacientes oncológicos com longo período de internação. Einstein. 2010;8(2):221-7. 


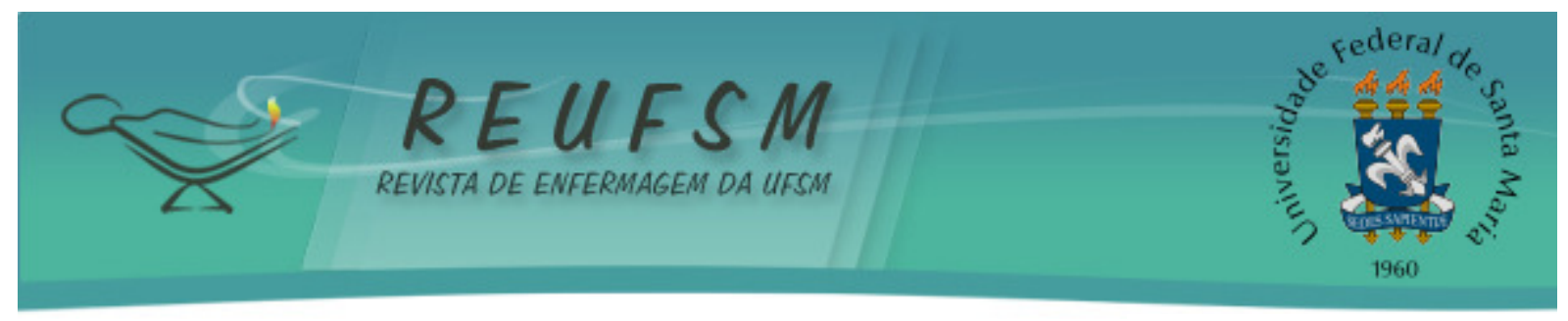

11. Rezende AM, Santos PP, Cerqueira ACM, Viana JL, Moderna CM. A criança e o adolescente com câncer em Casa de Apoio: projetando vivências. Rev SBPH. 2013;16(1):3-32.

12. Morais GSN, Costa SFG. Experiência existencial de mães de crianças hospitalizadas em Unidade de Terapia Intensiva Pediátrica. Rev Esc Enferm USP. 2009;43(3):639-46.

13. Moura CC, Resck ZMR, Dázio EMR. Atividades lúdicas realizadas com pacientes portadores de neoplasia internados em hospital geral. Rev Rene. 2012;13(3):667-76.

14. Brito TRP, Moreira DS, Resck ZMR, Marques SM. As práticas lúdicas no cotidiano do cuidar em enfermagem pediátrica. Esc Anna Nery Rev Enferm. 2009;13(4):802-8.

15. Gomes IP, Collet N. Distressful symptoms related to chemotherapy from the perspective of children: a qualitative research. Online Braz J Nurs [periódico na internet]. 2010 [acesso em 30 de mar 2015];9(2). Disponível em: http: //www.objnursing.uff.br/index.php/nursing/article/view/j.1676-

4285.2010.3045/683.

16. Lemos AC, Silva NCG. A função terapêutica da arte de contar histórias. Rev Digital. 2012;1(1):7-23.

17. Ceribelli C, Nascimento LC, Pacífico SMR, Lima RAG. A mediação de leitura como recurso de comunicação com crianças hospitalizadas. Rev Latino am Enferm. 2009;17(1):2-7.

Data de recebimento: $31 / 12 / 2015$

Data de aceite: 16/09/2016

Autor Principal: Silmara de Oliveira Silva

Endereço: Rua José Tomaz Filho, 215 - Paraíba CEP: 58398000 - Remígio-PB

Telefone: (81)9612-7472

Email: silmara_olyveira@hotmail.com 